\title{
Antimicrobial Potential of Crude Extracts of Thespesia populnea L. Flower on Multiple Drug Resistant Opportunistic Pathogens in HIV/AIDS
}

\author{
Jancy Varghese', S. Rajamani², Betty Daniel ${ }^{2}$
}

\section{Jancy Varghese', S. Raja- mani $^{2}$, Betty Daniel ${ }^{2}$}

'Research Scholar, Bharathiar University Coimbatore, Tamil Nadu, INDIA. ${ }^{2}$ Associate professor, $P G$ and Research Centre, St.Joseph's College Bangalore University, Karnataka INDIA.

${ }^{2}$ Head of the Department and Associate professor, PG and Research Centre, Karnataka St.Joseph's College Bangalore University, INDIA.

Correspondence

Jancy Varghese

Research Centre, St.Joseph's College, Langford road, Bangalore 560027, INDIA.

Phone No: 9535483217

E-mail: jansjc@gmail.com

History

- Submission Date: 05-01-2018;

- Review completed: 23-02-2018;

- Accepted Date: 23-02-2018

DOI : 10.5530/pj.2018.3.97

Article Available online

http://www.phcogj.com/v10/i3

\section{Copyright}

(C) 2018 Phcog.Net. This is an openaccess article distributed under the terms of the Creative Commons Attribution 4.0 International license.

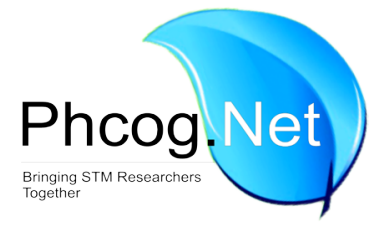

\begin{abstract}
Background: Thespesia populnea L. commonly known as tulip tree or 'portia' tree has been traditionally used for different illnesses. Thespesia populnea L. is considered to have high tannin content and have high therapeutic value. Purpose: The antifungal and antibacterial potential of T. populnea L. should be checked on multiple drug resistant opportunistic pathogens Pseudomonas aeruginosa and Candida albicans in HIV/AIDS patients. Methods: In order to check the effect of antibiotics antibiogram was studied with antibiotic sensitivity discs. To study the major factor affecting resistance test on formation of biofilm was done by tube and plate methods. The dried flower powder was subjected to sauxlaut hot extraction and the crude extract was analysed by Gas Chromatography and Mass Spectrophotoscopy. Antimicrobial potential of the ethyl acetate extract of the flower was checked by well agar diffusion, UV Spectrometry for growth rate and docking of selected compounds on the microbes of our study by the application of a software. Results: The tested extracts and the selected compounds have showed significant results in the antimicrobial activities against the opportunistic pathogens in human. Higher the concentration of the extracts better is the inhibition of microbes. Conclusion: The study provides a scientific rationale for the traditional use in the management of opportunistic pathogens which are multidrug resistant.
\end{abstract}

Key words: Antimicrobial, Antibiogram, Docking, Multidrug Resistant, Opportunistic Pathogens, Inhibition, Biofilm.

\section{INTRODUCTION}

Thespesia populnea $\mathrm{L}$ is also known as 'portia' tree, is a species of flowering plant under the family Malvaceae. It has a short, straight or crooked trunk. The flower is bell-shaped, 4-7 cm in length with five overlapping broad rounded petals. The leaves are applied locally in swollen joints for their anti-inflammatory effects and also for skin diseases, hepatitis, jaundice, ulcers, wounds, psoriasis, scabies, urinary tract infections, diabetes, cholera, cough, asthma and guinea worm infections. ${ }^{1}$ The barks and flowers possess astringent, hepatoprotective, antioxidant and anti-inflammatory activities in rats, and also supposed to improve the memory. ${ }^{2}$ The flowers of the same plant contained kaempferol, kaemperol-7-glucoside and gossypetin. ${ }^{3}$

Infectious diseases caused by bacteria, fungi, viruses and parasites are still a major threat to public health, despite the tremendous progress in human medicine. Their impact is particularly large in developing countries due to the relative unavailability of medicines and the emergence of widespread drug resistance. ${ }^{4}$ Numerous classes of antimicrobial agents have now become less effective as a result of the effective pressure of antimicrobial usage. Among the more important emerg- ing resistance problems are oxacillin resistance in staphylococci, penicillin resistance in streptococci, vancomycin resistance in enterococci (and eventually staphylococci), resistance to extended-spectrum cephalosporin and fluoroquinolones in Enterobacteriaceae, and carbapenem resistance in P.aeruginosa. ${ }^{5}$ In recent years, nosocomial infections caused by Pseudomonas aeruginosa have been recognized as an acute problem in hospitals due to its intrinsic resistance to many antibiotic classes and its capacity to acquire practical resistance to all effective antibiotics. ${ }^{6}$ The major cause of the resistance is due to the production biofilms. Pseudomonas aeruginosa is a gram negative bacteria and Candida albicans are the most prominent fungus occasionally associated with opportunistic diseases of humans. Considering the high costs of synthetic drugs and their various side effects, search for the alternative products from plants used in traditional system of medicine is justified. $^{\text {? }}$

This project aims to investigate antimicrobial properties and the presence of active phytochemical compounds in the flowers of T. populnea L. The extracts

Cite this article: Varghese J. Antimicrobial Potential of Crude Extracts of Thespesia populnea L. Flower on Multiple Drug Resistant Opportunistic Pathogens in HIV/AIDS. Pharmacog J. 2018;10(3):590-7. 
were prepared from dried powdered flowers using ethyl acetate. The extract was tested against $P$. aeruginosa and C. albicans for antimicrobial activity. It was observed that the microbes showed inhibition in growth when they were treated with the extract in different concentrations. This result suggests that T. populnea L. flower contains phytochemicals of medicinal properties.

\section{MATERIAL AND METHODS}

\section{Collection and isolation of opportunistic pathogens}

The clinical samples were collected by sterile collection swabs provided by Himedia and were cultured in a beef extract broth. When the culture was turbid inoculum was streaked in laminar air flow chamber on HiCrome Candida differential Agar (M1297A-Himedia, Mumbai) and, Pseudomonas Isolation Agar Base (M406-Himedia, Mumbai) in petri plates with sterile inoculation loop. Then the plates were sealed with klin wrap and kept in the incubator for $27^{\circ} \mathrm{C}$ for 24 to $48 \mathrm{~h}$ for fungus and $35^{\circ} \mathrm{C}$ for $24 \mathrm{~h}$ bacteria. The morphological identification of the colonies were done. Isolates were identified by standard microbial procedure (Gram staining, colonial morphology, catalase tests, and biochemical tests and molecular identification). The isolated colonies were stored in eppendorf tubes on nutrient agar base.

\section{Plant material and extraction}

T. populnea L. Flowers were collected from South India. The collected flowers were cut into small pieces and shade dried for 15 days. Once the flowers were completely dried they were ground into coarse powder and stored in the bottles. The coarse powder of the flowers was used as sample to obtain crude extracts using Ethyl acetate (EA). The sample was weighed exactly 30 grams and taken for extraction in Sauxlet apparatus. The solvent was measured $200 \mathrm{ml}$ for the extraction process. The Sauxlet apparatus were run for 3 days. The initial weight of the petri plate was determined. The crude extract obtained from extraction process was collected on to the petri plates and kept for air drying for 2 days. The air dried petri plates were weighed and the final weight of the petri plate was determined. The air dried extract was reconstituted with $10 \mathrm{ml}$ pure solvent from which the extract was obtained. It was then analysed phytochemically and was used for anti-microbial activity.

\section{Phytochemical prospection}

EA extract was tested for the presence of secondary metabolites ( alkaloids, carbohydrates, steroids, proteins, phenols, tannins, flavonoids, glycosides, gums, saponnins and terpenes) using standard procedures to identify the constituents as described by Sofawara (1993), Trease and Evans (1989), Harborne (1973) and Edeoga (2005).

\section{GC-MS analysis}

The crude extracts were submitted to GC-MS analysis and were found to contain a high number of metabolites. GC-MS chromatogram of the ethyl acetate extract of T. populnea L showed three major peaks has been identified after comparison of the mass spectra with NIST library, indicating the presence of three phytocomponents. Mass spectra of the separated components from the extracts were compared with the known components in the NSIT database.

\section{Antibiotic sensitivity test}

Antibiotic susceptibility test of biofilm producing bacteria was done on Muller Hinton agar (Himedia, Mumbai), using antibiotics discs obtained from Himedia Mumbai. Antibiotic susceptibility test was performed by using the Kirby-Buer disc diffusion technique according to CLSI guidelines. ${ }^{8}$
Hexa Antimyco-01 (HX104) and Pseudo-I (DE020) antibiotic disc rings were used for preparing antibiogram of the 30 strains of C. albicans and P. aeruginosa. These were inert flat circular rings (HX104) having 6 discs of $6 \mathrm{~mm}$ diameter on its projections of Amphotericin-B (AP) 100Unit, Clotrimazole (CC) $10 \mu \mathrm{g}$, Fluconazole (FLC) $25 \mu \mathrm{g}$, Itraconazole (IT) $10 \mu \mathrm{g}$, Ketoconazole (KT) $10 \mu \mathrm{g}$ and Nystatin (NS) 100Unit. DE020discs were coated with 12 antibiotics on its projections of Ceftazidime (CAZ)

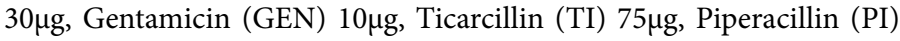

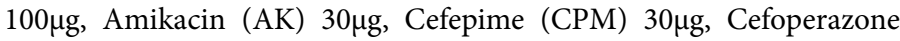
(CPZ) $75 \mu \mathrm{g}$, Ciprofloxacin (CIP) $5 \mu \mathrm{g}$, Levofloxacin (LE) $5 \mu \mathrm{g}$, Tobramycin

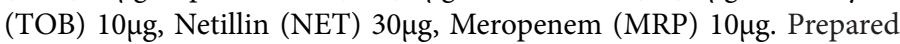
plates with Muller Hinton Agar $+2 \%$ Glucose $+0.5 \mathrm{mcg} / \mathrm{ml}$ Methylene Blue Dye (M1825) for carrying out susceptibility of antifungal discs for C. albicans. Prepared plates with Mueller Hinton Agar (M173/M1084) for rapidly growing aerobic organisms as per Bauer-Kirby Method. The medium in the plates was sterile and had a depth of about $4 \mathrm{~mm}$. Dipped a sterile non-toxic cotton swab and streaked the entire agar surface of the plates with the swab three times, turning the plate at $60^{\circ} \mathrm{C}$ angle between each streaking. Allowed the inoculum to dry for 5-15 min with lid in place. Deposited the rings at the center of the plate using sterile forceps. Incubated immediately at $35 \pm 2^{\circ} \mathrm{C}$ and examine after $16-18 \mathrm{~h}$. Measured the zones showing inhibition and record the diameters of the zones to the nearest millimeter.

\section{Biofilm test}

Biofilms are structured communities of bacteria embedded in a selfproduced matrix composed of exopolysaccharides, proteins, and extracellular DNA. Bacterial biofilms are notoriously known for their high resistance to antibiotics, disinfectant chemicals, and components of the innate and adaptive inflammatory defense system of the body. ${ }^{9}$ Microbes produce a biofilm matrix consisting of proteins, extracellular DNA, and polysaccharides that is integral in the formation of bacterial communities. ${ }^{10}$ Described by Christene et al, two qualitative methods are tube method and plate method which were used for biofilm formation. ${ }^{11}$

\section{Tube method}

A loop full of test organisms was inoculated in $10 \mathrm{ml}$ of nutrient broth. The tubes were incubated for $24 \mathrm{~h}$ at $37^{\circ} \mathrm{C}$ and $28^{\circ} \mathrm{C}$ respectively for Bacteria and fungus. After incubation tubes were decanted and washed with phosphate buffer saline ( $\mathrm{pH} 7.3$ ) and dried. Tubes were then stained with crystal violet $(0.1 \%)$. Excess stain was washed with deionized water. Tubes were dried in inverted position. The scoring for tube method was done according to the results of the control strains. Biofilm formation was considered positive when a visible film lined the wall and the bottom of the tube. The amount of biofilm formed was scored as weak, moderate and high. The experiment was performed in triplicate.

\section{Congo red agar method}

Freeman et al have described a simple qualitative method to detect biofilm production by using Congo Red Agar (CRA) medium. ${ }^{12}$ CRA medium was prepared with brain heart infusion broth) BHI) (Oxoid, UK) $37 \mathrm{~g} / \mathrm{L}$, sucrose 50 g/L, Agar No.1 (Oxoid, UK) 10 g/L. First Congo read stain was prepared as a concentrated aqueous solution and autoclaved (121C for $15 \mathrm{~min}$ ), separately from the other medium constituents. Then it was added to the autoclave BHI agar with sucrose at 55C.CRA plates were inoculated with test organism and incubated at $37 \mathrm{C}$ for $24 \mathrm{~h}$. Black colonies with dry crystalline constituency indicated biofilm production. The experiment was performed in triplicate. True negatives are those which were non biofilm producers by both methods. Sensitivity and specificity of TM was $73 \%$ and $92.5 \%$ respectively. For CRA method sensitivity and specificity remained low and were $11 \%$ and $92 \%$ respectively. 


\section{Antimicrobial assay by well diffusion}

The selected bacterial cultures (C. albicans and P. aeruginosa) were swabbed on sterile Muller Hinton agar using sterile cotton swabs. Agar wells were prepared with the help of sterilized Cork borer with $10 \mathrm{~mm}$ diameter. Using a micropipette $150 \mu \mathrm{l}$ of different extract was added to the wells in the plate. The plates were incubated in an upright position at $37^{\circ} \mathrm{C}$ for $24 \mathrm{~h}$. The diameter of inhibition zone, using ampicillin as control, was measured and was recorded. (Figures 1 and 2)

\section{Spectrometry}

The crude extracts were tested against the microbe's $P$. aeruginosa and Candida albicans. Pure cultures of these micro-organisms were prepared a day before the experiment using nutrient broth. The extracts were serially diluted using nutrient broth. The dilutions were made up to $10^{-5}$ From each serially diluted extracts, $1 \mathrm{ml}$ was pipetted into the eppendorff tubes using micropipettes. To each of these test tube $0.5 \mu \mathrm{l}$ of the pure culture of the microbes were added. These tubes were kept in incubator

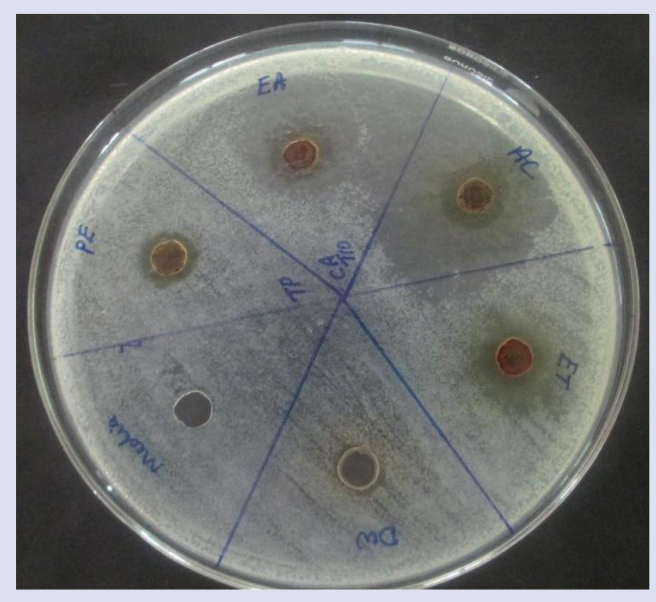

Figure 1: Antimicrobial assay by well diffusion on five flower extracts of T.populnea L. on C.albicans

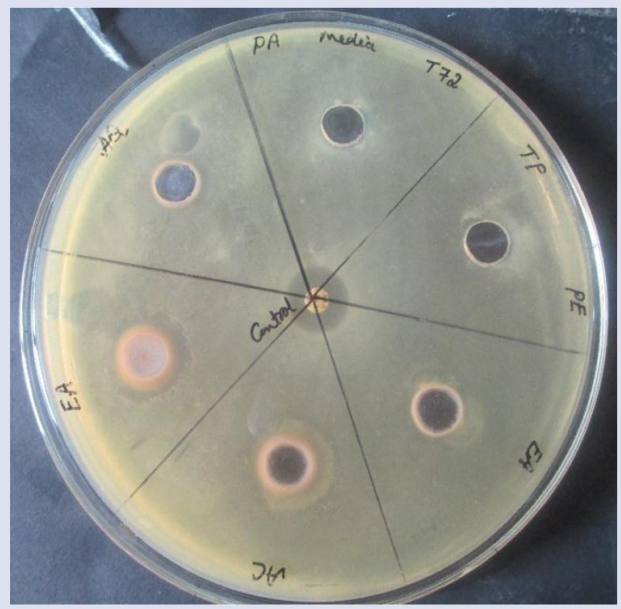

Figure 2: Antimicrobial assay by well diffusion on five flower extracts of T.populnea L on P.aeruginosa

PE-Petroleum ether, EA-Ethyl acetate, AC-Acetone, ET-Ethanol, DW-Distilled water, TP-Thespesia populnea L, CA-Candida albicans, PA- Pseudomonas aeruginosa, Control-Antibiotic at $37^{\circ} \mathrm{C}$. The incubation period was determined based on the doubling time of each of the microbes. Periodic absorbance of samples were measured at $650 \mathrm{~nm}$ by spectrophotometer (UV/VIS Spectrophotometer-SP-3000 Plus- Optima, Tokyo, Japan). Blank reading was set with nutrient broth. The rate of absorbance is directly proportional to the turbidity. Extract was used as standard. The turbidity of the culture was measured. The obtained values were depicted in the graphs.

\section{Protein docking}

Macromolecular docking is the computational modelling of the quaternary structure of complexes formed by two or more interacting biologicalmacromolecules. ${ }^{13}$

Protein-protein complexes are the most commonly attempted targets of such modelling, followed by protein-nucleic acid complexes. ${ }^{14}$ Here biologically active compounds Fatty acid decanoic acid or capric acid was selected for protein docking on HIV integrase (PDB ID: 1QS4). Antibacterial compound Capric acid or Deccanoic from Ethyl acetate extract showed maximum effects with minimum energy binding force. The selected compounds were subjected to protein docking using auto doc 4.A docking study of target proteins involved in antibacterial mechanisms was performed to extend the knowledge on standard antibiotics to herbal compounds which reported antibacterial activity. ${ }^{15}$ The minimum energy binding showed its maximum affinity of selected plant compound to the bacterial and fungal proteins.

Docking studies were performed by the application of software Autodock 4 on selected three compounds in order to evaluate their affinity to bacterial and viral proteins that are known targets for some antibiotics with different mechanism of action: inhibitors of cell wall synthesis, inhibitors of protein synthesis, and inhibitors of nucleic acids synthesis.

Docking was performed on antibacterial, and antifungal compounds Capric acid or Deccanoic acid. In the present work, the knowledge on target proteins of standard antibiotics was extended to antimicrobial plant compounds. ${ }^{15}$ The minimum energy binding shows its maximum affinity of selected plant compound to the bacterial and viral proteins. Overall, it seems that for capric acid the main mechanism of the action is the inhibition of cell wall synthesis.

The protein of $C$. albicans selected for this study was Dihydrofolate reductase (PDB ID: 4HOE). It is an enzyme that reduces dihydrofolate (FH2) to tetrahydrofolate (FH4) and is the sole source of FH4, thus having a central role in the maintenance of cellular process including acid biosynthesis, mitochondrial and chloroplast protein synthesis and amino acid metabolism etc. ${ }^{16}$ So in C. albicans it is providing a basis for the design of potentially selective inhibitors. The protein of $P$. aeruginosa selected for this study was, beta-lactamase (PDB ID: IFOF) - provide resistance to $\beta$-lactam antibiotics such as penicillins, cephalosporins, cephamycins etc. and it is also an enzyme which catalyses the biosynthesis, for P.aeruginosa. ${ }^{17}$ Targeting this protein inhibit the progression of infections by $P$. aeruginosa in host.

\section{RESULTS AND DISCUSSION}

\section{Collection and isolation of opportunistic pathogens}

Clinical samples collected from HIV/AIDS patients showed the presence of both microbes of our study in one or the other samples. Strains of C. albicans were found maximum in throat lesions and skin lesions (88\% and $47 \%$ ). Strains of $P$. aeruginosa was found mostly in vaginal swabs and throat swabs (47\% and 35\%). C. albicans were found as green colonies on HiCrome Candida differential Agar (M1297A) and P. aeruginosa was found as greenish yellow colonies on Pseudomonas Isolation Agar Base (M406). (Table 1) 


\section{Antibiogram}

Antibiogram shows the resistance patterns of 36 strains of C. albicans. All six antibiotics were resisted by various strains. Out of 36 strains tested all were resistant to almost all antibiotics (89\%- AMP, 11\%, 94\%- CL, 94\%-FL, 100\%-IT, 93\%-KET, 92\%-NS). Antibiogram shows the resistance patterns of 30 strains of $P$.aeruginosa. All 12 antibiotics were resisted by various Strains. (93\%- CEF, 77\%- GEN,76\%-TI, PI- 33\%, 93\%-AMK, 100\%- CEF, 87\%-CIF, 90\%-CEP, 87\%-LE, $93 \%$ TB, $97 \%$ NT, CEF, CEP and MEP showed intermediate results of $98 \%$ to $100 \%$. (Figure 3,4,5 and 6)

\section{Phytochemical prospection and metabolites analysis}

Phytochemical tests indicated the presence of alkaloids, phenolic flavonoids, flavanoids, carbohydrates, anthraquinones and tannins. GCMS chromatogram in Figure 5 shows 19 compounds in various peaks. Among 33 compounds analysed from ethyl acetate extract of flowers, 2-phenylpropionic acid and Octadecatrienoic acid were with maximum peaks as 16.44 and 17.267 respectively. 12 compounds are found to be with biologically active( Table 2), among which Capric acid or decanoic acid of 6.137 retention time (area 194795, \% of area 0.33 )found to be with maximum pharmaceutical use. The name, molecular weight and structure of the components of the test materials were determined. Out of 27 compounds reported from ethyl acetate extract biologically active compounds are 1-(3,6,6-Trimethyl-1,6,7,7a-tetrahydrocyclopenta\{c\}pyran1 -yl) with Rt 10.332, dodecanoic acid with Rt 10.399, Germacrene-d with Rt 11.5, loliolide with Rt 14.978, hexadecenoic acid palmitic acid with Rt 12.766, and falcarinol with Rt 17.165.

\section{Antimicrobial assay by well diffusion}

\section{Well diffusion assay}

The clear zones around the wells showed the inhibiting effects of the extracts on the microbes. (Figure 5 and 6). C. albicans showed maximum inhibition zone of 23-26mm and P. aeruginosa showed inhibition zones of $11 \mathrm{~mm}$ to $13 \mathrm{~mm}$ when they were treated the ethyl acetate extract. Ketamycin which was used as standard for C.albicans showed 11-15mm inhibition zone. Ampicilin was used as control for P. aeruginosa showed 11-15 $\mathrm{mm}$ inhibition. (Figures 1 and 2)

Table 1: Frequency of isolated organisms from ear (E), skin(S), and vaginal (V) swabs of HIV patients.

\begin{tabular}{ccccccccc}
\hline Species & $(\mathrm{N}=18)-\mathrm{E}$ & $\%$ & $(\mathrm{~N}=\mathbf{1 8})-\mathrm{S}$ & $\%$ & $(\mathrm{~N}=64)-\mathrm{V}$ & $\%$ & $(\mathrm{~N}=110)-\mathrm{T}$ & $\%$ \\
\hline Candida albicans & 10 & $38 \%$ & 7 & $88 \%$ & 9 & $23 \%$ & 46 & $47 \%$ \\
Candida tropicalis & 0 & & 1 & $13 \%$ & 7 & $11 \%$ & 1 & $2 \%$ \\
$\begin{array}{c}\text { Pseudomonas } \\
\text { aeruginosa }\end{array}$ & 2 & $11 \%$ & 2 & $11 \%$ & 21 & $47 \%$ & 38 & $35 \%$ \\
\hline
\end{tabular}

Table 2: Bioactive compounds identified from GC-MS analysis of ethyl acetate flower extract of $T$. populnea L.

\begin{tabular}{|c|c|c|c|}
\hline SI No & Compound Name & Structure & Function \\
\hline 1 & 2-cyclopenten 1- one,2-hydroxy & & Toxic \\
\hline 2 & Glycerin & & $\begin{array}{c}\text { Eye Disorders, Cerebral Edema, Vasodialator, Constipation, } \\
\text { Vehicle for Other Medications }\end{array}$ \\
\hline 3 & Benzoic acid & & $\begin{array}{l}\text { Constituent of Whitfield's ointment which is used for } \\
\text { the treatment of fungal skin, benzoic acid is also a major } \\
\text { ingredient in both tincture of benzoin and Friar's balsam, }\end{array}$ \\
\hline 4 & Isoamyl acetate & 0 & Used to test the effectiveness of respirators or gas masks \\
\hline 5 & Propanedioic acid phenyl & & $\begin{array}{c}\text { surgical adhesive, Heart Disease, Fibromyalgia, Skin-Care } \\
\text { Benefits }\end{array}$ \\
\hline 6 & Tetradecanoic acid & & Effective at causing the liver to synthesize cholesterol \\
\hline 7 & Decanoic acid ( capric acid) & & Has strong antiviral and antimicrobial properties \\
\hline
\end{tabular}




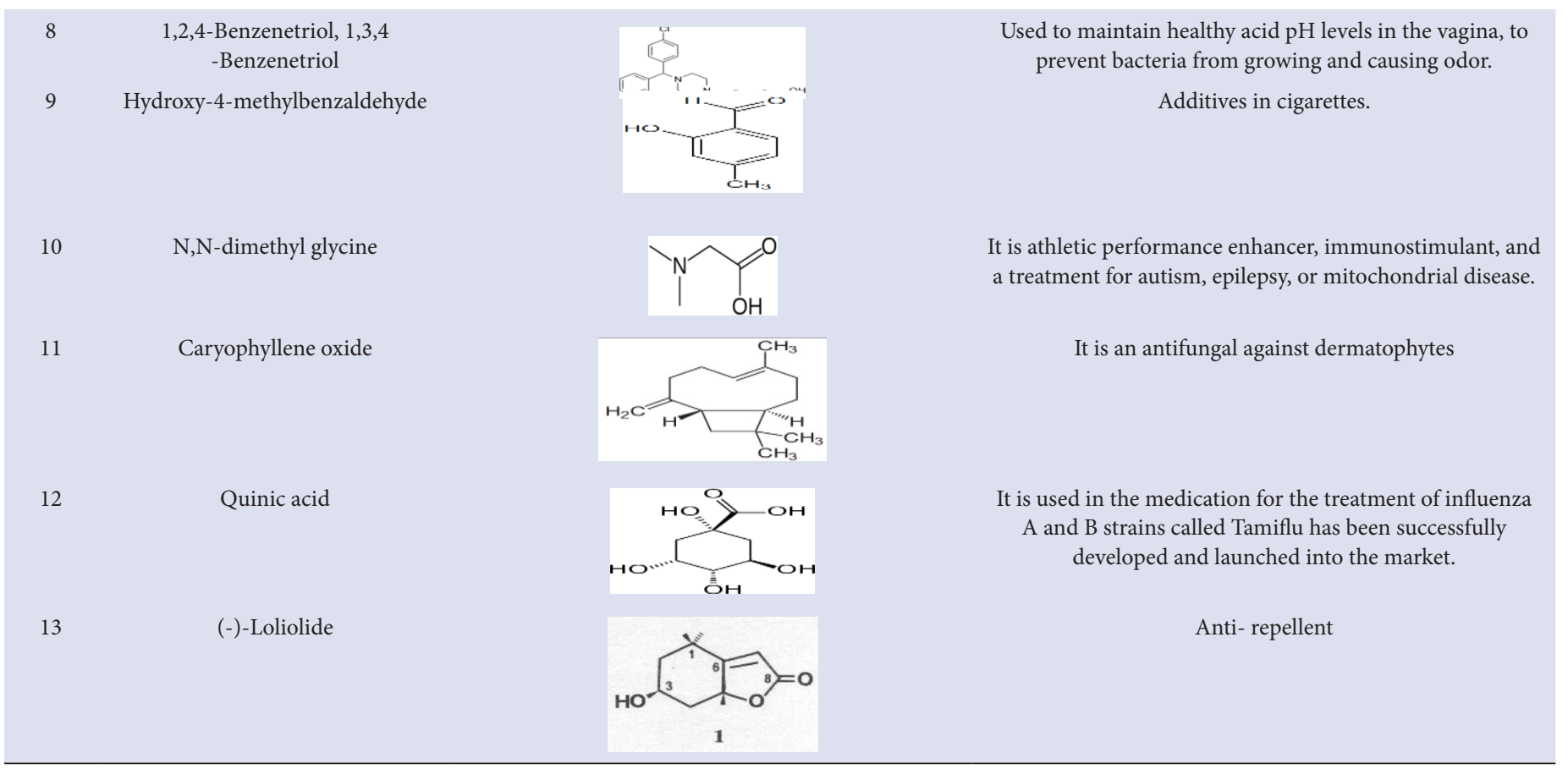

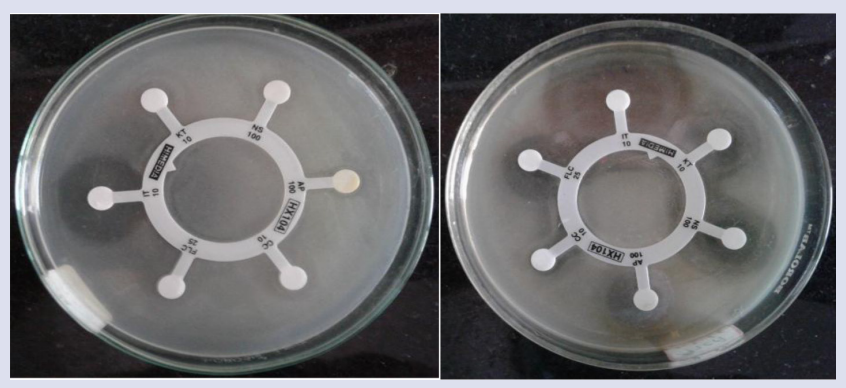

Figure 3: Antibiotic sensitivity test of C. albicans.

\section{Biofilm}

There were similarities and differences found in both TM and CRA methods. True negatives are those which were non-biofilm producers by both methods indicated as absent. Biofilm formation was found to be strong, moderate and weak in both methods.(Figure 7) C. albicans(CA) showed responses in all specificity (TM-Strong- $40 \%$, Moderate- $47 \%$, Weak-7\% and absent-6\%.CRA-Strong-17\%, Moderate-50\%, Weak-23\% and absent-10\%). P. aeruginosa also showed responses in all specificity (TM-Strong-56\%, Moderate- $44 \%$, weak- $0 \%$ and absent-0\%. CRAStrong- $0 \%$, moderate- $67 \%$, weak- $22 \%$ and absent-11\%).Compared to the results of CRA method TM results showed more results of sensitivity and specificity in case of both the organisms.

\section{Docking}

Protein docking results are expressed in terms of binding energy. Binding energy is released when a molecule associates with a target leading to a lowering of the overall energy of the complex. The release in the binding energy also compensates for any transformation of the ligand from its energy minimum to its bound conformation with the protein. Greater the energy released on binding of a ligand to the protein, a greater will be the propensity of the ligand to associate with that protein. The selected compound were subjected to protein docking using auto doc 4. The protein docking results of Capric acid all shown negative binding energy.

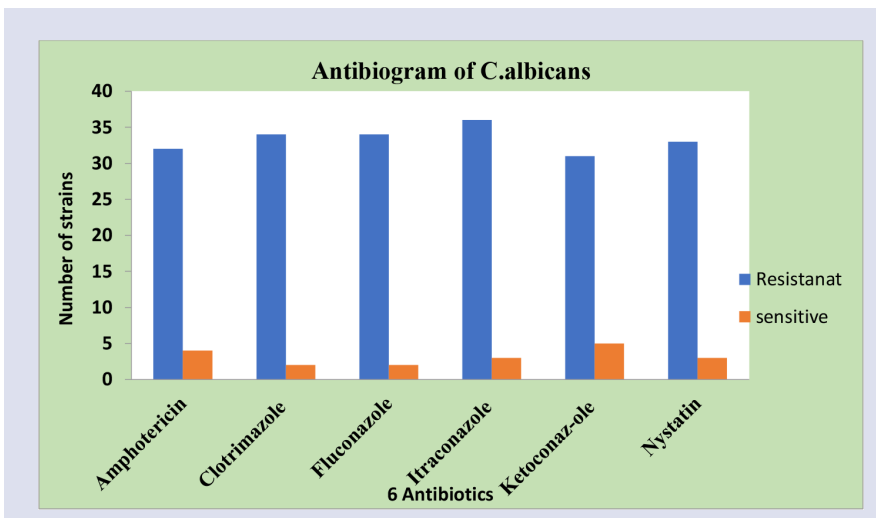

Figure 4: Antibiotic sensitivity test of C. albicans.

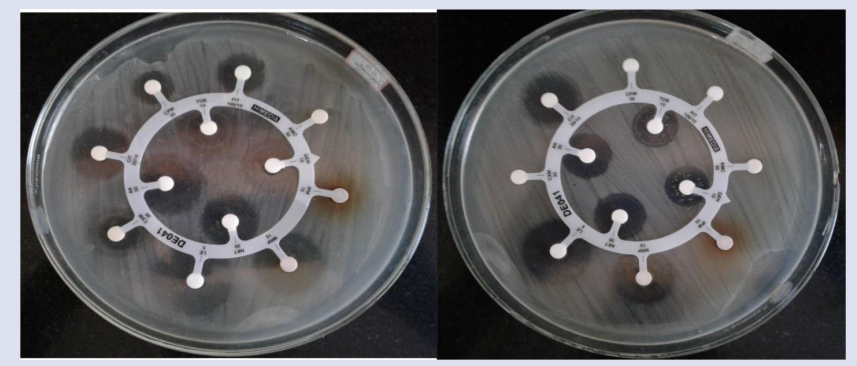

Figure 5: Antibiotic sensitivity test of $P$. aeruginosa. 


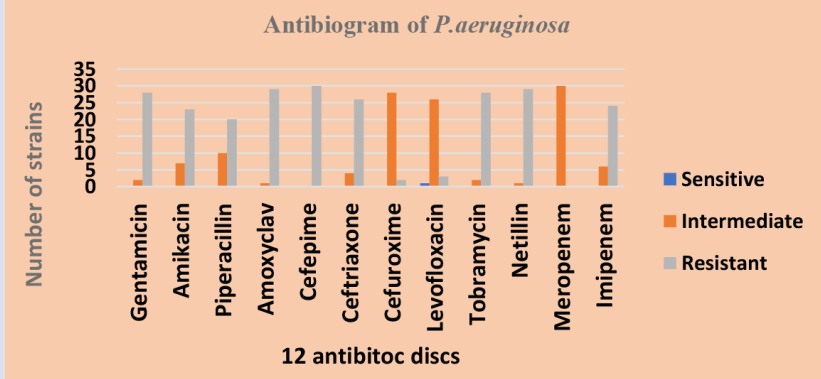

Figure 6: Antibiotic sensitivity test of $P$. aeruginosa.

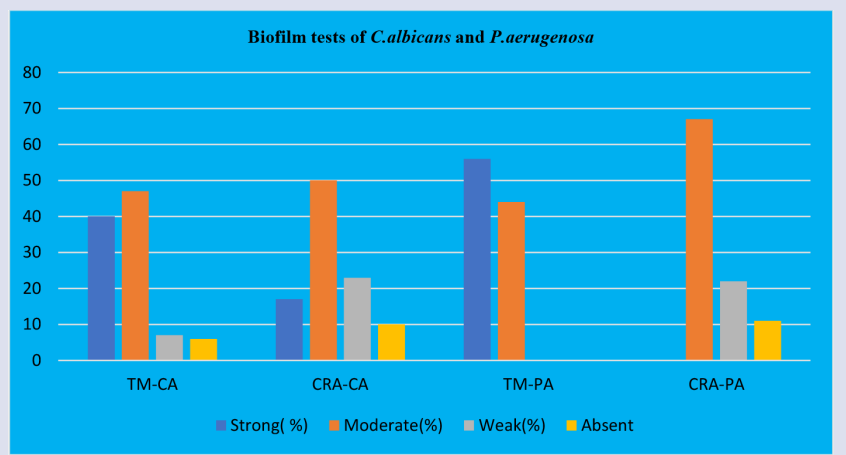

Figure 7: Biofilm test by TM and CRA method.

If the binding energy is negative then the ligand bind spontaneously without consuming energy and more stable the complex. Antibacterial, antifungal and antiviral compound Capric acid or Deccanoic from ethyl acetate extract showed maximum effects with minimum energy binding force. (Tables 3 and 4, Figure 8-11).

\section{Spectrometry}

When C. albicans and P. aeruginosa were grown in different dilutions of the ethyl acetate extract. The growth rate of C. albicans was increased at first and then decreased after some incubation period. The growth was more inhibited in the first, second and third dilutions. P. aeruginosa showed maximum inhibition in higher concentrations of extract. Gradually the growth rate increased as the concentration of the extract decreased. (Figures 12 and 13).

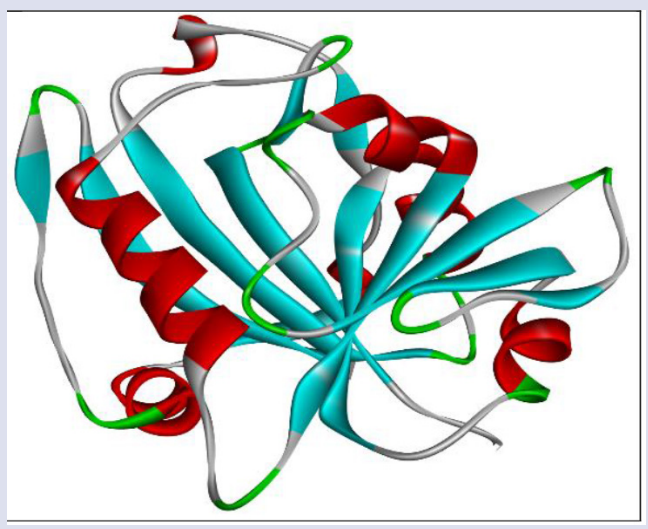

Figure 8: Chemical structure of Dihydrofolate reductase

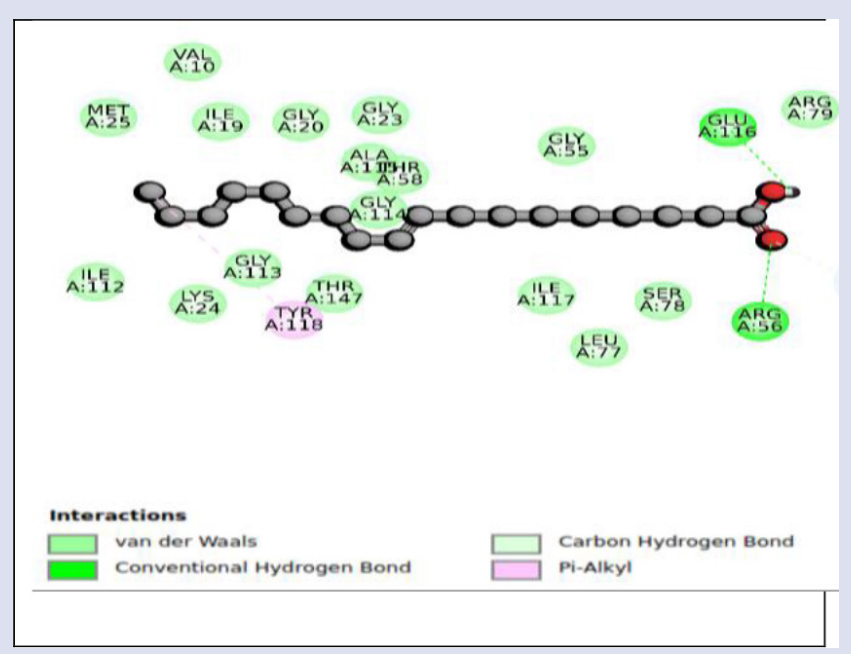

Figure 9: Interaction of the enzyme of C.albicans

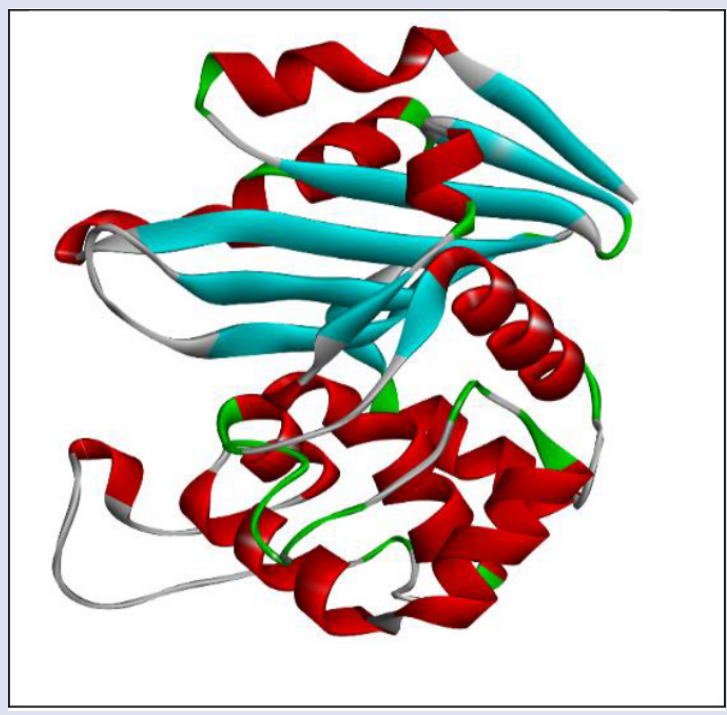

Figure 10: Chemical structure of Beta lactamase

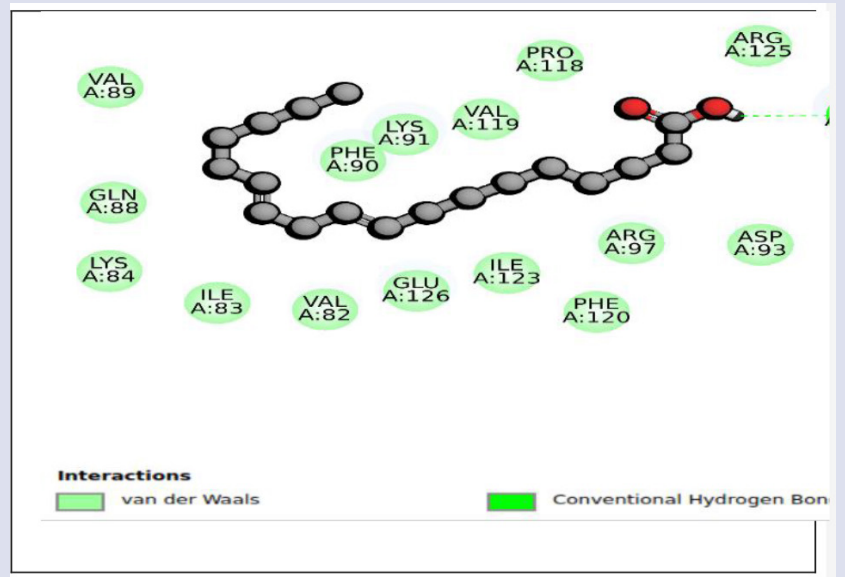

Figure 11: Interaction of the enzyme of P.aeruginosa 
Table 3: Binding Energy Score (C.albicans).

\begin{tabular}{cccccccc}
\multicolumn{3}{c}{$\begin{array}{c}\text { Rank Sub-rank Run Binding energy } \\
\text { (Kcal/mol) }\end{array}$} & \multicolumn{4}{c}{$\begin{array}{c}\text { Rank Sub-rank Run Binding energy } \\
\text { (Kcal/mol) }\end{array}$} \\
\hline 1 & 1 & 14 & -6.44 & 8 & 2 & 2 & -4.46 \\
2 & 1 & 11 & -5.97 & 9 & 1 & 10 & -5.45 \\
3 & 1 & 7 & -5.96 & 10 & 1 & 9 & -5.25 \\
4 & 1 & 5 & -5.87 & 11 & 1 & 13 & -5.09 \\
5 & 1 & 4 & -5.68 & 12 & 1 & 1 & -4.91 \\
6 & 1 & 8 & -5.63 & 13 & 1 & 3 & -4.54 \\
7 & 1 & 6 & -5.45 & 14 & 1 & 12 & -4.53 \\
\hline
\end{tabular}

Antifungal Test: (Best binding energy) is - 6.44

Table 4: Binding Energy Score (P.aeruginosa).

\begin{tabular}{cccccccc}
\hline \multicolumn{3}{c}{$\begin{array}{c}\text { Rank Sub-rank Run Binding energy } \\
\text { (Kcal/mol) }\end{array}$} & \multicolumn{4}{c}{$\begin{array}{c}\text { Rank Sub-rank Run Binding energy } \\
\text { (Kcal/mol) }\end{array}$} \\
\hline 1 & 1 & 14 & -4.98 & 4 & 2 & 2 & -4.68 \\
1 & 2 & 5 & -4.78 & 5 & 1 & 6 & -4.74 \\
2 & 1 & 11 & -4.93 & 6 & 1 & 4 & -4.67 \\
2 & 2 & 12 & -4.39 & 6 & 2 & 7 & -4.33 \\
3 & 1 & 8 & -4.87 & 7 & 1 & 3 & -4.64 \\
3 & 2 & 1 & -4.81 & 8 & 1 & 13 & -4.63 \\
4 & 1 & 0 & -4.76 & 9 & 1 & 15 & -4.39 \\
& & & & 10 & 1 & 10 & -3.84 \\
\hline
\end{tabular}

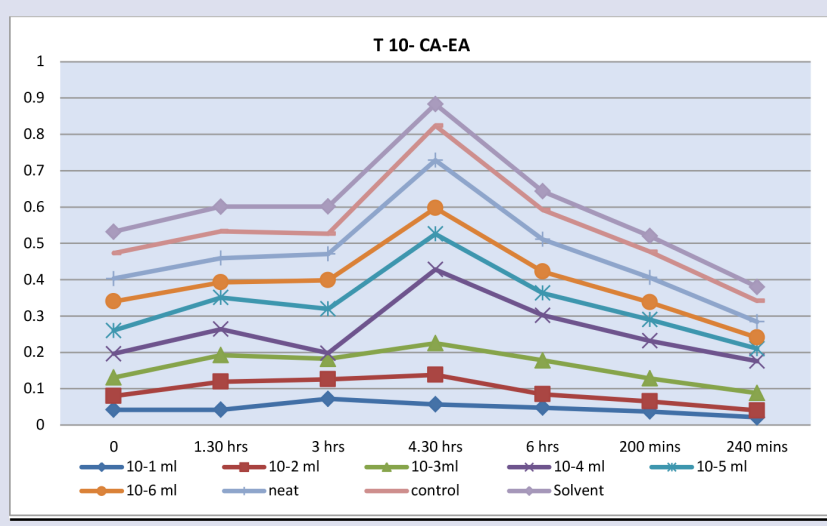

Figure 12: Growth rate of C.albicans with Ethyl acetate extract of T.populnea L. flower.

\section{CONCLUSION}

The study showed the antimicrobial effects of ethyl acetate extract of T. populnea L flower. Various experiments performed on this extract and its compounds proved could be used in replacement therapy for the multiple drug resistant opportunistic pathogens most commonly found in HIV/AIDS positive. Many of the antibiotics are resisted by human pathogens and results showed the inhibitory factors of opportunistic infection causing pathogens in HIV/AIDS patients. Data from results reveal the great potential of plant for therapeutic uses. It could be noted that there is future scope for the evaluation of T. populnea $\mathrm{L}$ flower extract for in vivo pharmacological activities.

\section{ACKNOWLEDGEMENT}

Our sincere thanks to the management of St. Joseph's College, Bangalore, for providing all laboratory facilities. We express our gratitude to

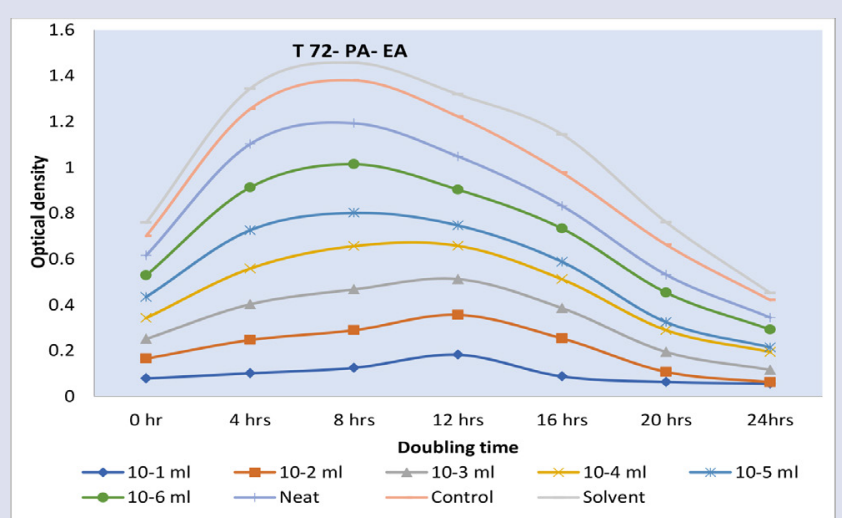

Figure 13: Growth rate of P.aeruginosa with Ethyl acetate extract of T.populnea L. flower

all those who have contributed their might for the carrying out of this project.

\section{ABBREVIATIONS USED}

HIV: Human Immuno Deficiency Virus; AIDS: Acquired immune Deficiency Syndrome.

\section{CONFLICT OF INTEREST}

The authors declare no conflict of interest.

\section{REFERENCES}

1. Neethu S. Kumar L, Neethu Simon. In vitro Antimicrobial Activities and Phytochemical Analysis of Crude Leaf Extracts of Thespesia Populnea. International Journal of Current Research in Life Sciences. 2016;(05):566-8

2. Abdul RS, Elyas M, Tara Thomas, Zahoorullah S. MD. Antidiabetic effect of some medicinal plants bark, leaf and seed extracts in streptozotocin induced diabetic 
rats.International Journal of Advanced Research. 2016; 4(12):1142-5.

3. Nandhini S, Radhika V, Manisha S, Anusha Jeslin V. Phytochemical Studies and Antimicrobial Compounds from Fruit of Thespesia Populnea (L . Asian Journal of Pharmaceutical and Clinical Research. 2017; 10(4):309.

4. Das MP, Banerjee A, Anu. Comparative study on in vitro antibacterial and antifungal properties of five medicinal plants of west bengal. Asian Journal of Plant Science and Research, Pelagia Research Library. 2013;3(3):107-1.

5. Beceiro A, Tomás M, Bou G. Antimicrobial resistance and virulence: a successful or deleterious association in the bacterial world. Clinical Microbiology Reviews. 2013;26(2):185-230

6. Fazeli H, Akbari R, Moghim S, Narimani T, Arabestani MR, Ali R. Ghoddousi - Pseudomonas aeruginosa infections in patients, hospital means, and personnel's specimens. Journal of Research in Medical Sciences. 2012;17(4):332.

7. Moon A, Khan A, Wadher B. Antimicrobial potential of the leaves of thespesia populnea (linn), sol. Ex corr leaves and its corresponding callus against drug resistant isolates. Indian Journal of Natural Products and Resources. 2010;1(4):444-9

8. Saffari N, Ahrabi SS, Ali AA, Hemami RM. A Comparison Of Antibiotic Disks From Different Sources On Quicolor And Mueller-Hinton Agar Media In Evaluation Of Antibacterial Susceptibility Testing. Iranian Journal of Microbiology. 2016;8(5):307.

9. Olivares E, Badel-Berchoux S, Provot C, Jaulhac B, Prévost G. Thierry Bernardi, et al. The biofilm ring test: a rapid method for routine analysis of pseudomonas aeruginosa biofilm formation kinetics. Journal of Clinical Microbiology. 2016;54(3):657-61.

\section{ABOUT AUTHORS}

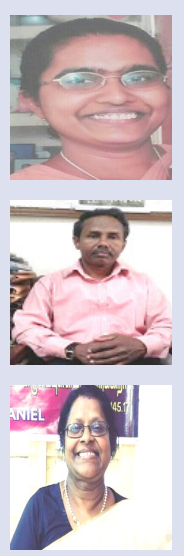

Sr. Jancy Varghese, Research Scholar, Bharathiar University Coimbatore, Tamil Nadu, India University, Karnataka, India.
10. Dominique HL, Christopher JJ, Daniel JW. Bacterial Extracellular Polysaccharides In Biofilm Formation And Function. American Society for Microbiology. 2015;3(3). doi:10.1128/microbiolspec.MB-0011-2014.

11. Hassan A, Usman A, Kaleem F, Omair M, Khalid A, Muhammad Iqbal Evaluation of different detection methods of biofilm formation in the clinical isolates. Brazilian Journal of Infectious Diseases. 2011;15(4):305-11.

12. AfreenishHassan A, JavaidUsman J, Kaleem F, Omair M, Khalid A, Iqbal Evaluation Of Different Detection Methods of Biofilm Formation in the Clinical Isolates. Brazilian Journal of Infectious Diseases. 2011;15(4):305-11.

13. Macromolecular docking, Wikipedia, the free encyclopedia, Asian month https://en.wikipedia.org/wiki/Macromolecular_docking.

14. Vijayakumar B, Kumar PD. Molecular Docking Studies-A Review. International Journal of Medicinal Chemistry and Analysis. 2012;151(7):1474-87.

15. Alves MJ, Froufe HJ, Costa AF, Santos AF, Oliveira LG, Osório SR. Docking studies in target proteins involved in antibacterial action mechanisms: extending the knowledge on standard antibiotics to antimicrobial mushroom compounds. Molecules. 2014;19(2):1672-84. Pubmed.

16. Whitlow M, Howard AJ, Stewart D, Hardman KD, Kuyper LF, Baccanari DP. X ray crystallographic studies of candida albicans dihydrofolate reductase. High resolution structures of the holoenzyme and an inhibited ternary complex. The Journal of Biological Sciences. 1997;272(48):30289-98. PubMed.

17. Godfrey AJ, Bryan LE. Resistance of pseudomonas aeruginosa to new lactamase b-lactamase. Antimicrobial Agents and Chemotherapy. 1984 Oct; 26(4): 485-8.

\section{SUMMARY}

- T.populnea L. flower extract significantly inhibited the opportunistic pathogens C.albicans and Paeruginosa which cause major infections in HIV/AIDS patients. The antimicrobial properties of this plant indicate that this herbal medicine could be a promising alternative therapy to the use of antibiotics which are resisted by these microbes which are major threats to humanity.

Dr. S. Rajamani, Msc, MPhil, and PhD from Madras University, India. He is specialized in Mycology and Microbiology and a faculty of St.Joseph's College, Bangalore University, Karnataka, India.

Dr.Betty Daniel, Msc, MPhil, PhD from National Centre for Biological Sciencesv(NCBS), Bangalore. Specialized in Molecular Cancer Biology and the Head of the Department of Botany, St.Joseph's College, Bangalore

Cite this article: Varghese J. Antimicrobial Potential of Crude Extracts of Thespesia populnea L. Flower on Multiple Drug Resistant Opportunistic Pathogens in HIV/AIDS. Pharmacog J. 2018;10(3):590-7. 